\title{
Estudio de la producción científica de las publicaciones en México referentes a la catalogación de documentos: 1990-2009
}

\author{
Adelina Clausó GARCíA \\ Universidad Complutense de Madrid. Facultad de Ciencias de la Documentación \\ aclauso@pdi.ucm.es \\ Antonio CARPALlO BAUTISTA \\ Universidad Complutense de Madrid. Facultad de Ciencias de la Documentación \\ acarpallo@eubd.ucm.es
}

Recibido: septiembre 2011

Aceptado: noviembre 2011

Resumen: En este artículo se hace un balance de la producción científica mexicana de monografías, artículos de revistas, congresos y sus comunicaciones, así como Tesis, Tesinas e Informes académicos referentes a la catalogación de documentos entre los años 1990-2009, a partir de los registros encontrados en la base de datos INFOBILA, en la base de datos CLASE, en el OPAC de la Biblioteca Nacional de México, en el OPAC de la biblioteca del CUIB y en la revista Investigación Bibliotecológica. Se estudia la fecha de publicación, la autoría, el género, los lugares y entidades editoras, entre otros aspectos de interés que posibilitan un acercamiento a esta área del conocimiento.

Palabras clave: Catalogación; Producción Científica; Bibliometría

Study of the scientific production of the publications in Mexico refered to the cataloguing of documents: 1990-2009

\begin{abstract}
This article evaluates Mexican scientific monograph production, magazine articles, congresses and its communications, as well as Thesis, Dissertations and Academic Reports about Cataloguing of documents between 1990 and 2009, from the registers found in INFOBILA database, in CLASE database, in the OPAC of National Library of Mexico, in the OPAC of CUIB library and in Investigación Bibliotecológica magazine. The publication date, authorship, genre, publishing places and entities are studied, among others interest aspects that make possible an approach to this area of the knowledge.
\end{abstract}

Keywords: Cataloguing; Scientific Production; Bibliometrics 


\section{INTRODUCCIÓN ${ }^{1}$}

El objetivo fundamental de este estudio es dar una visión global de las publicaciones aparecidas en México durante los años 1990-2009, referente a la catalogación de documentos, materia esta relevante dentro de los estudios de biblioteconomía y documentación.

Iniciamos este trabajo haciendo una breve exposición de la historia de estos estudios en México, a fin de demostrar cómo desde sus inicios esta materia ha estado, y sigue estando presente en todos y cada uno de los planes de estudios, lo que se ha traducido por una parte en la producción científica de publicaciones y por otra en la realización de trabajos tales como Informes académicos, Tesinas y Tesis.

Los estudios bibliotecarios aparecieron por primera vez en México en el año 1945 cuando se creó la Escuela Nacional de Biblioteconomía y Archivonomía, dependiente de la Secretaría de Educación Pública, elevándose a rango universitario en 1956 fecha en la que nace, en la Facultad de Filosofía y Letras dentro de la Universidad Nacional Autónoma de México (UNAM), el Colegio de Bibliotecología ${ }^{2}$. Los estudios se inician con el nivel de Maestría en Biblioteconomía, estableciéndose en 1958 los niveles de licenciatura y de maestría en Biblioteconomía y Archivonomía. En 1966 se modificaron los planes de estudio así como también el nombre del Colegio que desde ese momento pasó a denominarse Colegio de Bibliotecología y Archivonomía. El citado Colegio tiene como objetivo fundamental formar profesionales capaces de planear, organizar y administrar bibliotecas, centros de información y centros de documentación. La columna vertebral sobre la cual giran las actividades de los licenciados es satisfacer las necesidades de información de los distintos tipos de comunidades para lo cual tienen que seleccionar, adquirir y organizar diferentes tipos de materiales. Desempeñan sus actividades en bibliotecas, centros de documentación, centros de información, redes de información, empresas proveedoras de servicios de información y bases de datos, así como en sistemas nacionales e internacionales de información.

El plan de estudios vigente de Licenciatura en Bibliotecología y Estudios de la Información fue aprobado por el Consejo Técnico en su sesión ordinaria del 8 de

${ }^{1}$ Este trabajo es producto de una comunicación presentada en el mes de abril de 2010 con motivo del VII Seminario Hispano-Mexicano celebrado en México D.F. y organizado por el CUIB.

${ }_{2}$ Arriola Navarrete, O. Panorama general de la educación bibliotecaria en México. ACIMED 2009; 19 (3). Disponible en http://bvs.sld.cu/revistas/aci/vol19 3 09/aci03309.htm. [Consultado 05/02/2010] 
febrero del 2002 y puesta en práctica el 16 de agosto de 2002 a partir del año escolar 2003; consta de 51 asignaturas, 360 créditos y una duración de cuatro años. ${ }^{3}$

Además se pueden cursar los programas de Maestría y Doctorado en Bibliotecología y Estudios de la Información a través de la Facultad de Filosofía y Letras y del Centro Universitario de Investigaciones Bibliotecológicas (CUIB).

Ocho instituciones más del país ofertan la carrera en sus distintos grados de Licenciatura o Maestría:

- Universidad Autónoma Nuevo León (UANL) Facultad de Filosofía y Letras. Estudios: Licenciatura en Bibliotecología y Ciencias de la Información. http://www.uanl.mx/oferta/licenciatura/facultades/ffyl/lic_bibliotecologia.html

- Universidad Autónoma San Luis de Potosí (UASLP) Escuela Ciencias de la Información. Estudios: Licenciado en Bibliotecología.

http://portal.uaslp.mx/Spanish/Administracion/ServEsc/admisiones/Document s/nuestrascarreras0910.pdf

- Universidad Autónoma de Chihuahua Facultad de Filosofía y Letras. Estudios: Licenciado en Ciencias de la Información. http://www.uach.mx/academica_y_escolar/carreras/planes/2008/11/04/faculta d_de_ciencias_de_la_informacion/

- Universidad Autónoma de Chiapas (UACH) Facultad de Humanidades. Estudios: Licenciado en Bibliotecología y Gestión de la Información. http://secacad.unach.mx/images/stories/humanidades/bibliotecologia.pdf

- Universidad Autónoma del Estado de México Facultad de Humanidades. Estudios: Licenciado en Ciencias de la Información Documental. $\mathrm{http}: / / \mathrm{www} \cdot d$ ep.uaemex.mx/mapas/dspmapcur.asp?pe=11\&tpo=g

- Universidad Autónoma Metropolitana Unidad Xochimilco. Estudios: Maestría en Gestión de la Información.

http://psa.xoc.uam.mx/posgrados/mgi.html

- Instituto Tecnológico de Estudios Superiores de Monterrey, Universidad Virtual del Tecnológico de Monterrey. Estudios: Maestría en Ciencias de la Información.

http://www.ruv.itesm.mx/portal/promocion/oe $/ \mathrm{m} / \mathrm{mik} / \mathrm{homedoc} . \mathrm{htm}$

- Escuela Nacional de Biblioteconomía y Archivonomía. Dependiente de la Dirección General de Educación Superior Universitaria. Estudios: Licenciatura en Biblioteconomía.

http://www.enba.sep.gob.mx/new800x600/licbiblioteco/indexlicbiblio.htm

${ }^{3}$ Universidad Nacional Autónoma de México. Facultad de Filosofía y Letras. Colegio de Bibliotecología. http://www.filos.unam.mx/LICENCIATURA/bibliotecologia/colegio.html. [Consultado 05/02/2010] 
De las universidades mencionadas, en relación con la materia de Catalogación, las que cuentan con más créditos en la impartición de la misma son: Universidad Nacional Autónoma de México (UNAM), Universidad Autónoma Nuevo León (UANL) y Escuela Nacional de Biblioteconomía y Archivonomía.

\section{METODOLOGÍA}

Para la elaboración de este estudio se han consultado diferentes fuentes, exclusivamente producidas por instituciones mexicanas ${ }^{4}$, como la base de datos INFOBILA $^{5}$ del Centro Universitario de Investigaciones Bibliotecológicas (CUIB) de la Universidad Nacional Autónoma de México, la base de datos CLASE ${ }^{6}$ mantenida por la UNAM, el catálogo automatizado de la Biblioteca Nacional de México ${ }^{7}$, el catálogo de la Biblioteca del CUIB y la web de la revista Investigación Bibliotecológica ${ }^{8}$, empleando como estrategia de búsqueda la palabra clave Catalogación,

${ }^{4}$ Somos conscientes de que autores mexicanos han publicado, por ejemplo, en revistas internacionales, pero no se han consultado bases de datos de este tipo ya que lo que se pretende es estudiar la producción científica de los autores mexicanos en México, tal y como se indica en el título del trabajo.

${ }^{5}$ Esta base de datos, creada por el Centro Universitario de Investigaciones Bibliotecológicas (CUIB) de la UNAM en 1985, tiene como objetivo recopilar la producción latina de trabajos relacionados con las bibliotecas, archivos y documentación. El sistema empleado para la gestión de los 12.000 registros aproximadamente es Micro CDS/ISIS bajo formato MARC. La información puede recuperarse por varios campos: autor, título, país, fechas, descriptores, etc. Los registros ofrecen una descripción formal de los documentos, junto a un resumen y unos descriptores extraídos del Tesauro Latinoamericano en Bibliotecología y Ciencias de la Información. http://cuib.unam.mx/infobila/indice.html [consultado: 10/02/2010]

${ }^{6}$ Esta es una base de datos bibliográfica, creada y mantenida por la Universidad Nacional Autónoma de México (UNAM), que contiene cerca de 200.000 registros bibliográficos de artículos, ensayos, reseñas de libro, revisiones bibliográficas y otros documentos publicados en más de 1.200 revistas de América Latina y el Caribe, especializadas en ciencias sociales y humanidades. http://132.248.9.1:8991/F/-/?func=find-b-0\&local_base=CLA01 [consultado: $10 / 02 / 2010]$

${ }^{7}$ La Biblioteca Nacional, fundada en 1867, tiene como objetivo la conservación del patrimonio bibliográfico de México, siendo la mayor biblioteca con más de 1.250 .000 libros y documentos. http://biblional.bibliog.unam.mx/bibn/ [consultado: 10/02/2010]

${ }^{8}$ Investigación Bibliotecológica es una revista científica mexicana de periodicidad cuatrimestral, editada por el CUIB, donde se incluyen artículos evaluados por investigadores y docentes de reconocido prestigio nacional e internacional, así como su Consejo Editorial. Cada artículo cuenta con resumen en español e inglés, palabras clave y otros elementos de identificación; también se incluyen reseñas bibliográficas sobre trabajos publicados de la especialidad. La revista está incluida en el índice de Revistas Mexicanas Científicas y Tecnológicas del Consejo Nacional de Ciencia y Tecnología (CONACYT) y es indizada en Social Sciences Citation Index, LISTA, LISA, ISA, CLASE e INFOBILA. http://cuib.unam.mx/revista1.htm [consultado: $10 / 02 / 2010]$ 
para la localización de las monografías, artículos y comunicaciones a congresos, y el término geográfico México para el lugar, en el periodo comprendido entre 1990 y 2009.

También se han incluido las referencias de los trabajos (informes académicos, tesinas, tesis) generados por los alumnos de las titulaciones de Bibliotecología durante el mismo periodo, ya que se exigen para la obtención del título de Licenciado, como se indica, por ejemplo, en el reglamento de la UNAM, aprobado el 28 de octubre de $2004^{9}$. Dichas referencias han sido extraídas de la base de datos CLASE.

Una vez identificados los diferentes tipos documentales, monografías, artículos y literatura gris, se extrajeron los siguientes datos a través de los registros bibliográficos: año de publicación, autoría, tipo de autoría, género, entidades editoras y descriptores. En relación a estos últimos destaca la gran diversidad de términos empleados para indizar el mismo contenido, así por ejemplo Catalogación de Acervos Videográficos, Normas, Videocintas y Videograbaciones; aunque también nos hemos encontrado términos generales como Catalogación y otros mucho más específicos como Catalogación de Materiales Audiovisuales.

A la vista de la diversidad de términos empleados en la indización de los documentos seleccionados, y concluida la captura de datos, se procedió a la normalización con el fin de obtener una uniformidad en los campos, agrupando bajo un mismo término todos aquellos que podían tener el mismo significado, así por ejemplo bajo el término Catalogación se agruparon todos los relacionados con Normas, Reglas de catalogación, Análisis documental, Asientos corporativos, etc., siguiendo la misma metodología con el resto de los descriptores.

Posteriormente se procedió al análisis de los datos y a su difusión mediante figuras y tablas.

\section{RESULTADOS}

En este apartado se estudia un total de 111 publicaciones, de las cuales 56 corresponden a monografías, 20 a artículos de revista, 5 a contribuciones a congresos, jornadas, etc., y por último 30 que corresponden a trabajos de alumnos que bien pueden ser informes académicos, tesinas o tesis.

\footnotetext{
${ }^{9}$ En este punto debemos aclarar que a diferencia de los trabajos de investigación presentados en las universidades españolas para la obtención del actual título de grado, equivalente al de Licenciado en México, los alumnos pueden optar a distintas modalidades de trabajos denominados informes académicos, tesinas, tesis.
} 


\subsection{MONOGRAFÍAS}

\subsubsection{AÑOS DE PUBLICACIÓN}

En la siguiente figura podemos observar la frecuencia de publicación de monografías durante el periodo estudiado.

Fig. 1. Producción por años

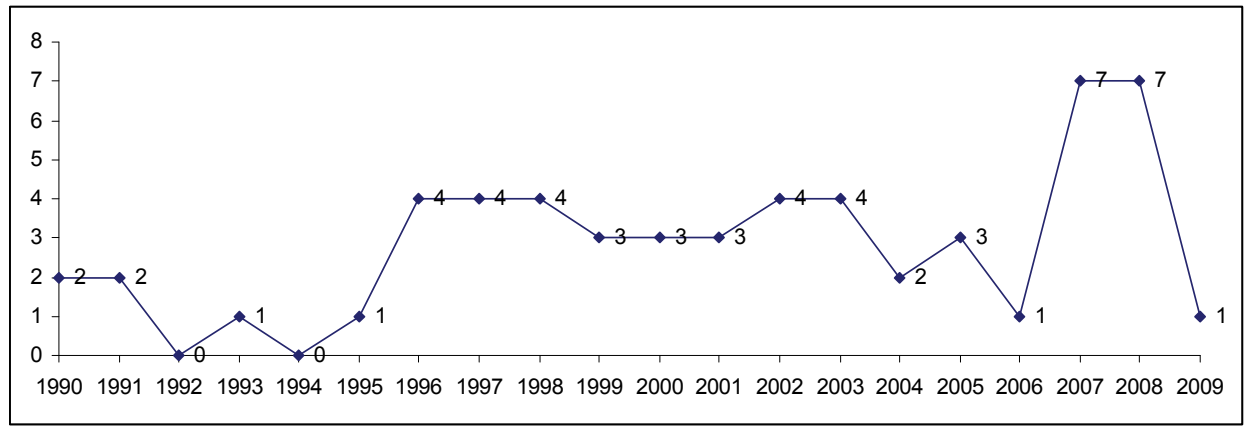

Destaca por encima de todos el periodo 2007-2008 con siete monografías por año, apreciándose una regularidad en la producción, entre dos y cuatro monografías, en el periodo que abarca de 1996 a 2005.

\subsubsection{AUTORÍA}

En las cincuenta y seis monografías localizadas han intervenido un total de veinte autores, de los cuales el $80 \%$ son autores únicos y el resto con autoría compartida, observándose por tanto que predomina la autoría única, y confirmándose así los estudios que muestran la tendencia de la individualidad dentro del Área de Humanidades y Ciencias Sociales frente a la colectividad en el Área de Ciencias Técnicas y de la Salud, esto mismo será posteriormente aplicable, como veremos, a los artículos de revista.

El promedio entre el número de autores y trabajos es de 1,25.

Tabla I. Número de autores participantes por trabajo

\begin{tabular}{|c|c|}
\hline Firmantes por trabajo & Número de casos \\
\hline $\mathbf{1}$ firma & $\mathbf{1 6}$ \\
$\mathbf{2}$ firmas & $\mathbf{2}$ \\
$\mathbf{3}$ firmas & $\mathbf{2}$ \\
Más de 3 firmas & $\mathbf{0}$ \\
\hline Total & 20 \\
\hline
\end{tabular}


Fig. 2. Coautoría

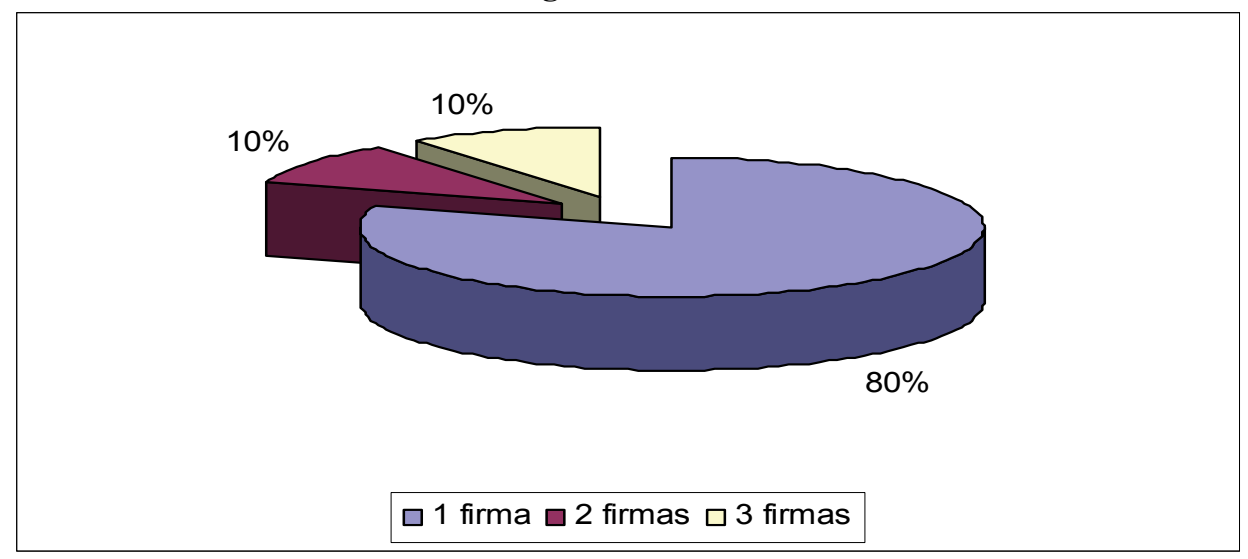

\subsubsection{TIPO DE AUTORÍA}

En este apartado analizamos si la responsabilidad recae en una entidad o en una persona, así como si es el responsable total de la obra o participa como prologuista, compilador, traductor, colaborador, coordinador, etc. Sólo encontramos una publicación con autoría corporativa realizada por el Comité Técnico de Normalización Nacional de Documentación.

En total encontramos veinticinco autoridades de las cuales quince han participado como autores, dos como autores y traductores y/o compiladores, y ocho han participado sólo con otro tipo de responsabilidad.

Fig. 3. Tipo de autoría

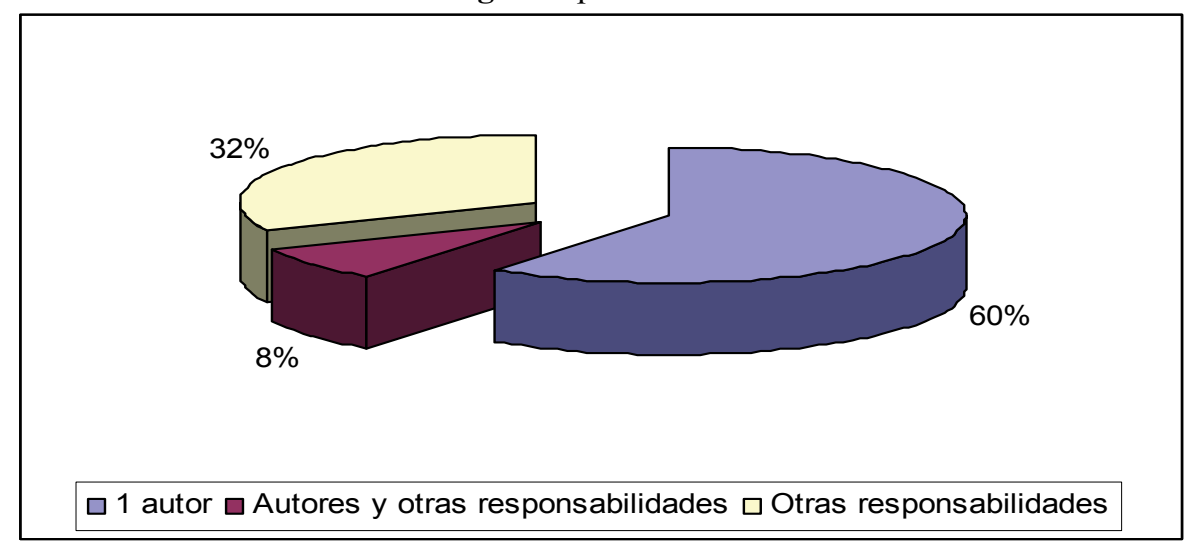


Entre los autores más productivos se encuentran Concepción Barquet Téllez, Gloria Escamilla González, Carlos Benigno García López, Roberto Garduño Vera, Filiberto Felipe Martínez Arellano, Catalina Naumis Peña y Emma Norma Romero Tejeda. Otras responsabilidades, entre las que destacan la traducción, y que aparecen con mayor frecuencia corresponden a Gloria Escamilla González y Filiberto Felipe Martínez Arellano.

Destacar a Roberto Garduño Vera y Gloria Escamilla González con más de una publicación como autores únicos, esta última también resalta por su labor como traductora; subrayar a Carlos Benigno García López, Concepción Barquet Téllez, Catalina Naumis Peña, Emma Norma Romero Tejeda y Filiberto Felipe Martínez Arellano que han participado como autores en más de una publicación tanto como responsables únicos como con autoría compartida, además Martínez Arellano también ha figurado como compilador y traductor.

\subsubsection{GÉNERO}

Del total de las veinticinco autoridades (autores y otros responsables), se evidencia una clara distancia entre ambos géneros, ya que un $60 \%$ son mujeres y un $40 \%$ son hombres.

Tabla II. Distribución de autores según su género

\begin{tabular}{|c|c|c|}
\hline Género de los responsables & $\mathrm{N}^{\mathrm{o}}$ de responsables & Porcentaje (\%) \\
\hline Hombre & $\mathbf{1 0}$ & $\mathbf{4 0} \%$ \\
Mujer & $\mathbf{1 5}$ & $\mathbf{6 0} \%$ \\
\hline Total & 25 & $100 \%$ \\
\hline
\end{tabular}

\subsubsection{LUGARES Y ENTIDADES EDITORAS}

En relación a los lugares de publicación, todas las obras han sido publicadas en México D.F. Entre las entidades editoras destaca la Universidad Nacional Autónoma de México (UNAM) con dieciséis publicaciones junto a la Escuela Nacional de Biblioteconomía y Archivonomía con tres y el Instituto Latinoamericano de la Comunicación Educativa (ILCE) con dos. Dentro de la UNAM han sido varias las entidades subordinadas que han participado como entidades editoras resaltando el Centro Universitario de Investigaciones Bibliotecológicas (CUIB) con cinco obras, el Instituto de Investigaciones Bibliográficas y la Dirección General de Bibliotecas ambas con cuatro; otras entidades dentro de la UNAM que han participado en la edición han sido la Facultad de Filosofía y Letras, la Escuela Nacional de Estudios Profesionales y la Secretaría de Servicios Académicos. Señalar que todas las entidades editoras pertenecen al ámbito público. 
Tabla III. Editoriales que más han publicado

\begin{tabular}{|l|c|}
\hline Entidades editoras & $\mathbf{N}^{\mathbf{0}}$ de monografías \\
\hline UNAM. CUIB & 5 \\
\hline UNAM. Instituto de Investigaciones Bibliográficas & 4 \\
\hline UNAM. Dirección General de Bibliotecas & 4 \\
\hline $\begin{array}{l}\text { Escuela Nacional de Biblioteconomía y Archivonomía } \\
\text { (ENBA) }\end{array}$ & 3 \\
\hline $\begin{array}{l}\text { Instituto Latinoamericano de la Comunicación Educa- } \\
\text { tiva (ILCE) }\end{array}$ & 2 \\
\hline
\end{tabular}

Fig. 4. Porcentaje de monografías publicadas por la UNAM y otras entidades

\begin{tabular}{|l|}
\hline UNAM \\
$\square$ Escuela Nacional de Biblioteconomía y Archivonomía (ENBA) \\
Instituto Latinoamericano de la Comunicación Educativa (ILCE) \\
$\square$ Dirección General de Bibliotecas \\
$\square$ Información Científica Internacional \\
$\square$ Red Nacional de Bibliotecas Públicas
\end{tabular}

\subsubsection{FORMATO DE LAS PUBLICACIONES E IDIOMA}

Todas las monografías las hemos encontrado en formato libro, no siendo todavía el soporte electrónico el vehículo elegido para la difusión de este tipo de contenidos. En cuando al idioma todas las obras están en español, aunque hay que destacar que tres fueron traducciones de habla inglesa, realizadas dos de ellas por Gloria Escamilla González y una por Filiberto Martínez Arellano.

\subsubsection{DESCRIPTORES}

Haciendo un análisis estadístico de los descriptores, el término más empleado ha sido Catalogación, como entrada inicial, junto a otras acepciones similares como Catalogación descriptiva, Descripción bibliográfica, Análisis Documental, Asientos corporativos (catalogación), Análisis bibliográfico que hemos agrupado 
dentro del término Catalogación. También destaca el empleo de descriptores referidos a la catalogación específica de los diversos materiales como Catalogación de archivos de ordenador, Catalogación de casetes, Catalogación de libros antiguos, Catalogación de manuscritos, Catalogación de materiales audiovisuales, Catalogación de publicaciones periódicas, Catalogación de materiales especiales. Dentro del epígrafe Catalogación no podemos olvidar otros términos como Catalogación analítica, Catalogación centralizada, Catalogación cooperativa y Catalogación automatizada, esta última muy relacionada con la Catalogación en línea, Conversión retrospectiva (catalogación), Metadatos y Formato MARC, así como el término Catalogación - Manuales, etc., y Catalogación descriptiva Reglas, ISBD y Normas.

Tabla IV. Descriptores más empleados (Catalogación)

\begin{tabular}{|l|c|}
\hline \multicolumn{1}{|c|}{ Descriptor } & $\begin{array}{c}\mathbf{N}^{\mathbf{0}} \text { de ocasiones em- } \\
\text { pleado }\end{array}$ \\
\hline Catalogación & 30 \\
\hline Catalogación automatizada & 12 \\
\hline Catalogación de videograbaciones & 4 \\
\hline Catalogación cooperativa & 4 \\
\hline Catalogación - Manuales, etc. & 3 \\
\hline Catalogación - Normas & 3 \\
\hline
\end{tabular}

También aparecen otros descriptores como Bibliotecas universitarias, Clasificación, Automatización de bibliotecas, Catálogos colectivos, Catálogos de autori$\mathrm{dad}$, junto a varios relacionados con la recuperación y acceso a la información como Acceso a la información, Acceso al documento, Búsquedas en línea, Recuperación de información, Servicios de información, Sistemas de almacenamiento y recuperación de la información y Unidades de información.

Tabla V. Descriptores más empleados (excepto Catalogación)

\begin{tabular}{|l|c|}
\hline \multicolumn{1}{|c|}{ Descriptor } & $\begin{array}{c}\mathbf{N}^{\mathbf{0}} \text { de ocasiones em- } \\
\text { pleado }\end{array}$ \\
\hline Acceso a la información & 12 \\
\hline Bibliotecas universitarias & 9 \\
\hline Clasificación & 6 \\
\hline Catálogos de autoridades & 5 \\
\hline Catálogos colectivos & 4 \\
\hline Control bibliográfico & 4 \\
\hline
\end{tabular}




\subsection{ARTÍCULOS DE REVISTA}

\subsubsection{AÑOS DE PUBLICACIÓN}

En la siguiente figura podemos observar la frecuencia de publicación de artículos de revistas, con un total de siete publicaciones, durante el periodo estudiado.

Fig. 5. Producción por años

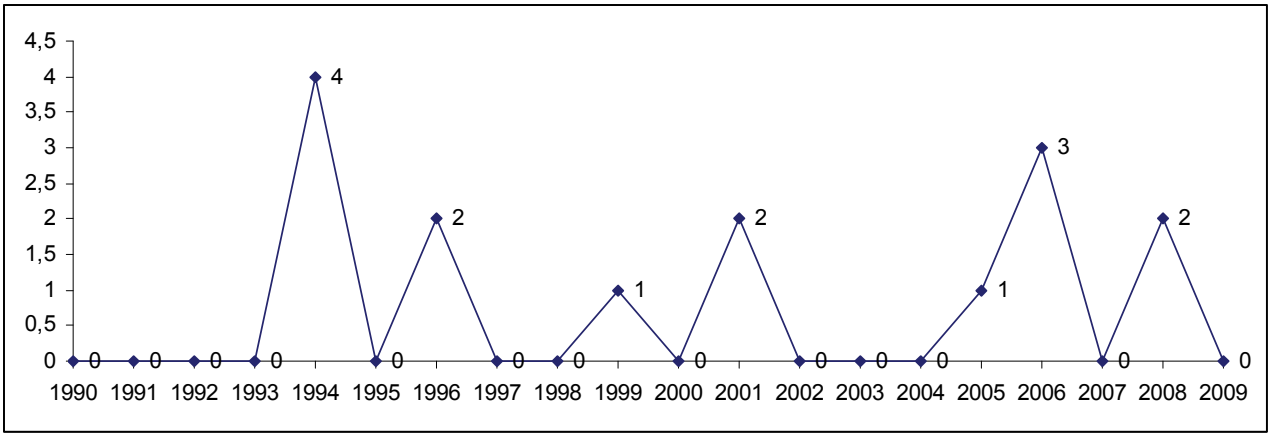

Los artículos identificados hacen un total de veinte, observándose que los años de mayor producción han sido 1994, con un total de cuatro trabajos, el año 2006 con tres trabajos y los años 1996, 1998, 2001, 2003, 2004 y 2008 con dos trabajos. El resto de los años han tenido un solo trabajo o incluso ninguno.

\subsubsection{AUTORÍA}

En los veinte artículos encontrados han intervenido un total de veintitrés autores, siendo el porcentaje de autores únicos de $75 \%$ y el resto con autoría compartida, observándose por tanto que predomina la autoría única. Es curioso observar que varios trabajos han sido firmados por tres, cuatro y cinco autores.

El promedio entre el número de autores y trabajos es de 1,15. 
Fig. 6. Coautoría

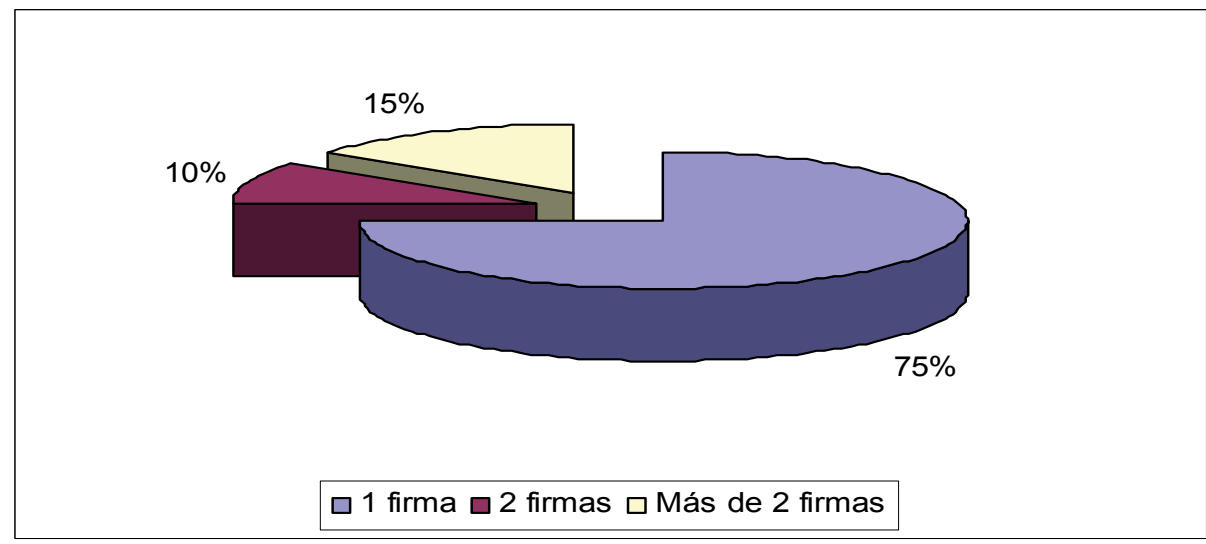

Tabla VI. Número de autores participantes por artículo

\begin{tabular}{|c|c|}
\hline Firmantes por trabajo & Número de casos \\
\hline $\mathbf{1}$ firma & $\mathbf{1 5}$ \\
\hline 2 firmas & $\mathbf{2}$ \\
\hline Más de 2 firmas & $\mathbf{3}$ \\
\hline Total & 20 \\
\hline
\end{tabular}

\subsubsection{TIPO DE AUTORÍA}

El total de los veintitrés autores, anteriormente mencionados, entre los más productivos destaca, por encima de todos, Ariel Alejandro Rodríguez García ${ }^{10}$ con cinco trabajos. El resto han realizado sólo un trabajo ya sea como autor único o de forma colectiva.

\subsubsection{GÉNERO}

Del total de los veintitrés autores no se evidencia diferencia entre ambos géneros ya que algo más del $52 \%$ son hombres y el $47 \%$ son mujeres.

${ }^{10}$ Este autor tiene entre otras titulaciones el Doctorado en Bibliotecología y Estudios de la Información, Maestría y Licenciatura en Bibliotecología, todos realizados en la Facultad de Filosofía y Letras de la UNAM. Su línea de investigación se centra en la normalización y sistematización de la información. Actualmente trabaja en temas relacionados con la catalogación descriptiva, la clasificación documental y los sistemas de recuperación de información. 
Tabla VII. Distribución de autores según género

\begin{tabular}{|c|c|c|}
\hline Género de los responsables & $\mathrm{N}^{\circ}$ de responsables & Porcentaje (\%) \\
\hline Hombre & $\mathbf{1 2}$ & $\mathbf{5 2 , 1 7} \%$ \\
Mujer & $\mathbf{1 1}$ & $\mathbf{4 7 , 8 2} \%$ \\
\hline Total & 23 & $100 \%$ \\
\hline
\end{tabular}

\subsubsection{REVISTAS MÁS PRODUCTIVAS}

Entre las revistas más productivas encontramos a Biblioteca universitaria ${ }^{11}$ con diez trabajos publicados, seguida por Investigación Bibliotecológica: archivonomía, bibliotecología e información, con cuatro artículos y con dos Hemera: Revista de ciencias bibliotecológica y de la información ${ }^{12}$. Las cuatro revistas restantes contienen un artículo sobre catalogación de documentos.

Fig. 7. Revistas más productivas

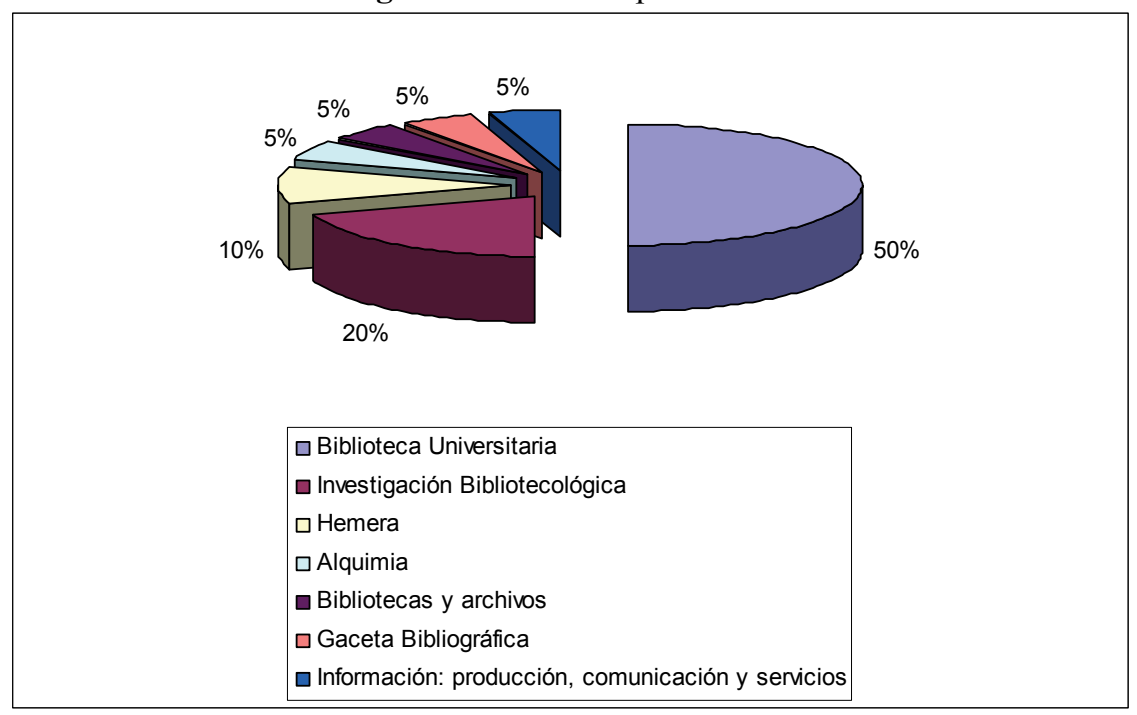

${ }^{11}$ Biblioteca universitaria está editada por la Dirección General de Bibliotecas de la UNAM desde 1986. Está indizada en las bases de datos CLASE, INFOBILA, COMPLUDOC y REDALYC. En su web se pueden consultar y descargar todos los artículos publicados en formato pdf. Su dirección es: http://www.dgbiblio.unam.mx/revista/Revista\%20online/ revista.html

${ }_{12}$ Hemera es una revista indizada por INFOBILA, Latindex, CLASE y Hela. En su web se puede consultar el índice acumulativo de los años 2003 y 2004. Su dirección es: http://hemera.galeon.com/ 


\subsubsection{AFILIACIÓN INSTITUCIONAL}

De los veintitrés autores mencionados, la mayoría pertenecen a la UNAM, destacando los adscritos a la Dirección General de Bibliotecas, al Centro Universitario de Investigaciones Bibliotecológicas, a la Facultad de Filosofía y Letras, al Instituto de Geología y al Instituto de Investigaciones Históricas.

Tabla VIII. Afiliación institucional de los autores

\begin{tabular}{|l|c|}
\hline \multicolumn{1}{|c|}{ Lugares de afiliación de los autores } & N $^{\mathbf{0}}$ de afiliaciones \\
\hline UNAM. Dirección General de Bibliotecas & 5 \\
\hline UNAM. CUIB & 5 \\
\hline UNAM. Facultad de Filosofía y Letras & 5 \\
\hline UNAM. Instituto de Geología & 3 \\
\hline Escuela Nacional de Biblioteconomía y Archivonomía & 2 \\
\hline UNAM. Instituto de Investigaciones Históricas & 1 \\
\hline El Colegio de México & 1 \\
\hline Instituto Nacional de Antropología e Historia & 1 \\
\hline
\end{tabular}

En este apartado se puede observar el alto número existente de autores pertenecientes a la UNAM, el 83\%, seguido de otros tres centros relacionados con la formación y la investigación.

Fig. 8. Tipología de las instituciones a las que pertenecen los autores de artículos

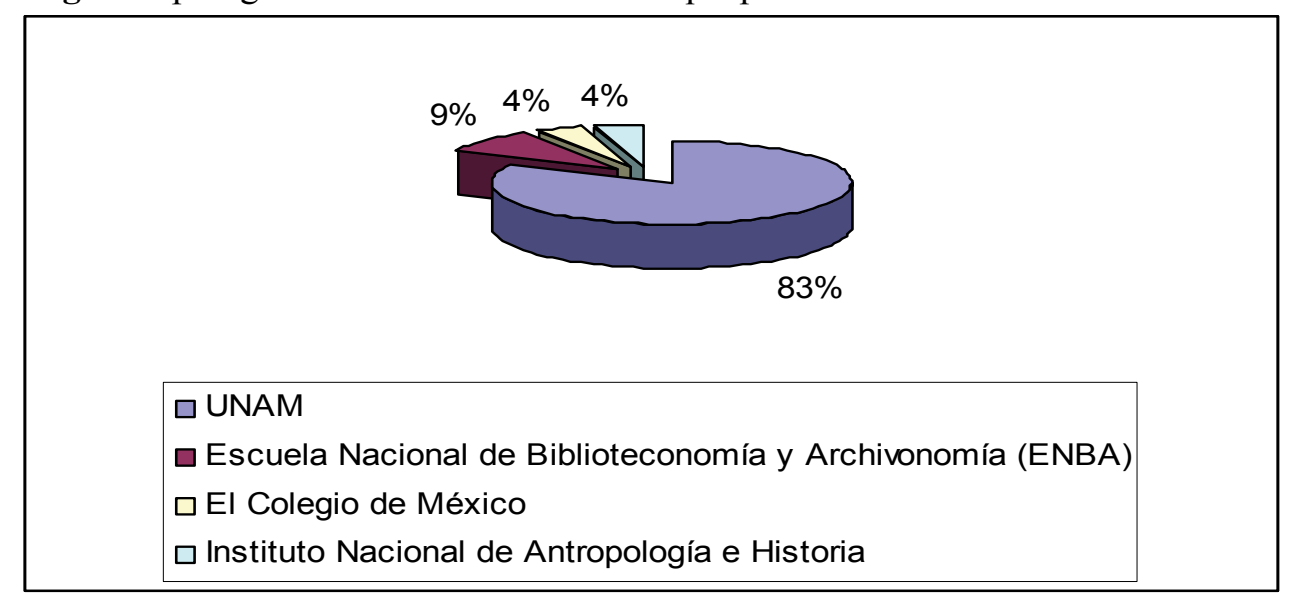

\subsubsection{DESCRIPTORES}

Al igual que el caso anterior encontramos que el descriptor más empleado ha sido Catalogación (15), seguido muy de lejos por el término Bibliotecas (5), Información electrónica (4), Normalización (3), Modelo FRBR (2), Reglas de 
Catalogación Angloamericanas (2), Catálogos de autoridad (2) y Control de calidad (2).

\subsection{CONGRESOS}

Dentro de este estudio también hemos querido incluir las actas y comunicaciones de entidades de carácter temporal tales como congresos, jornadas, etc., relacionadas con la catalogación. Comenzaremos por tanto indicando la publicación de actas de tres encuentros específicos sobre catalogación:

- Encuentro de Nacional Catalogación y Metadatos (3 ${ }^{\circ}$ 2008. México, D.F.)

- Encuentro Internacional de Catalogación $\left(2^{\circ}\right.$. 2006. México, D.F.)

- Encuentro Nacional de Catalogación y Metadatos ( $2^{\circ} .2007$. México, D.F.)

Los tres eventos fueron publicados por el CUIB participando como compiladores Filiberto Felipe Martínez Arellano y Ariel Alejandro Rodríguez García, dos de los autores más destacados tanto en la producción de monografías como de artículos de revista. Las actas de los tres eventos se publicaron al año siguiente de su celebración, es decir en 2007, 2008 y 2009.

En cuanto a las comunicaciones hemos localizado cinco específicas sobre catalogación realizadas por los siguientes autores:

- Garza González, Alejandro

- Martínez Arellano, Filiberto Felipe

- Pérez Pineda, Víctor Manuel

- Rodríguez García, Ariel Alejandro

- Wendler, Robin

Las comunicaciones se presentaron en los eventos que fueron organizados por el CUIB y por la Asociación Mexicana de Bibliotecarios A.C., dichos eventos fueron los siguientes:

- Coloquio de Investigación Bibliotecológica y de la Información $\left(20^{\circ}\right.$. 2002. México, D.F.)

- Coloquio Internacional de Investigación Bibliotecológica (16º . 1998. México, D.F.)

- Jornadas Mexicanas de Biblioteconomía (31․ 2000. Querétaro, Qro.)

- Seminario Nacional de Normatividad Archivística (1º ${ }^{\circ}$ 1996. México, D.F.)

Por último en los descriptores empleados para la indización de estas comunicaciones destaca el término Catalogación, junto a otros como Catalogación automa- 
tizada, Catalogación de documentos oficiales, Formato MARC, Metadatos, Normalización, Normas, Reglas de Catalogación Angloamericanas.

\subsection{LITERATURA GRIS (INFORMES ACADÉMICOS-TESINAS- TESIS)}

No queremos terminar este estudio sin incluir los trabajos (30) realizados por los alumnos de las diferentes titulaciones, dentro de los apartados Informes académicos, Tesinas y Tesis realizados durante el periodo estudiado entre 1990 y 2009.

Señalar que ninguno de los tres tipos de trabajos es inferior a otro y que se trata de diversas modalidades que los alumnos eligen libremente.

El 28 de octubre de 2004 se publicó en la Gaceta el Reglamento General de Exámenes de la UNAM y el Consejo Técnico de la Facultad de Filosofía y Letras de la UNAM modificó, en sus sesiones del 16 de marzo y del 27 de abril de 2007, la reglamentación para la presentación de este tipo de trabajos.

La Tesis es un trabajo cuyo objetivo es demostrar que el alumno cuenta con la formación para organizar, desarrollar y expresar de forma adecuada los conocimientos adquiridos en la disciplina correspondiente. Por tanto en la Tesis el alumno debe desarrollar un tema incluyendo información actualizada, con una estructura clara donde se incluya un índice y una bibliografía.

Se han encontrado ocho Tesis relacionadas con la Catalogación, defendidas seis de ellas en la UNAM, cuatro de estas en la Facultad de Filosofía y Letras, una en el Colegio de Bibliotecología y otra en la Escuela Nacional de Estudios Profesionales Acatlán; las otras dos Tesis fueron defendidas en la Dirección General de Educación Superior Universitaria, Escuela Nacional de Biblioteconomía y Archivonomía.

Indicar que de las ocho Tesis, tres fueron presentadas para la Maestría en Bibliotecología y Estudios de la Información en la Facultad de Filosofía y Letras de la UNAM, cuatro para Licenciaturas como Licenciado en Bibliotecología en el Colegio de Bibliotecología de la UNAM, Licenciado en Lengua y Literatura Hispánicas en la Escuela Nacional de Estudios Profesionales Acatlán de la UNAM y Licenciado en Biblioteconomía en la Escuela Nacional de Biblioteconomía y Archivonomía de la Dirección General de Educación Superior Universitaria. La última Tesis fue presentada en el Doctorado en Bibliotecología y Estudios de la Información de la Facultad de Filosofía y Letras, División de Estudios de Posgrado de la UNAM.

La Tesina es un trabajo monográfico de investigación cuyo objetivo, al igual que en la Tesis, es demostrar que el alumno cuenta con la formación necesaria en la disciplina correspondiente. Este trabajo deberá está bien organizado, estructurado junto a un índice y una bibliografía actualizada.

Hemos localizado seis Tesinas, cuatro defendidas en la Escuela Nacional de Biblioteconomía y Archivonomía, Dirección General de Educación Superior Uni- 
versitaria, y dos en el Colegio de Bibliotecología de la Facultad de Filosofía y Letras de la UNAM. Indicar que dos de ellas se presentaron para Licenciado en Bibliotecología, tres para Licenciado en Biblioteconomía y una para Licenciado en Archvonomía.

En cuanto al Informe académico pueden darse varios tipos: por actividad profesional, por artículo académico, de investigación, por elaboración comentada de material didáctico para apoyar la docencia, por servicio social y por trabajo de campo. Todos ellos deberán incluir la descripción de la tarea desarrollada, realizar una crítica del trabajo realizado, y adjuntar un índice y una bibliografía.

Este tipo de trabajos son los más numerosos con un total de dieciséis, de los cuales once se han presentado en el Colegio de Bibliotecología de la Facultad de Filosofía y Letras de la UNAM para la Licenciatura en Bibliotecología y cinco en la Escuela Nacional de Biblioteconomía y Archivonomía de la Dirección General de Educación Superior Universitaria para la Licenciatura en Biblioteconomía.

Fig. 9. Distribución de Tesis, Tesinas e Informes académicos

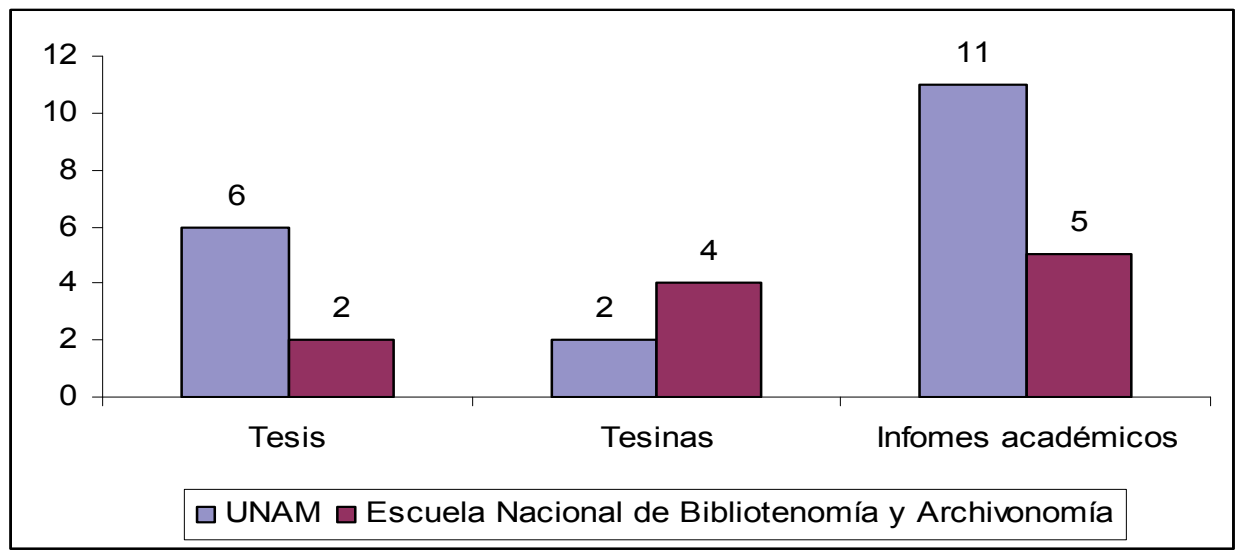

En cuanto a los asesores destacar a Filiberto Felipe Martínez Arellano y Esperanza Molina Mercado con tres direcciones y a Hugo Alberto Figueroa Alcántara con dos.

Otro aspecto a resaltar es la producción de este tipo de trabajos por años. Es curioso observar que la primera Tesis se defendió en 1991 aunque su producción en el periodo estudiado es baja (8). También es baja la producción de Tesinas (6) comenzando su presentación y defensa a partir de 2001. Sin embargo es mucho mayor la producción de Informes académicos (16) aunque también comenzaron su defensa a partir de 2000 siendo habitual la defensa de dos o tres trabajos desde 2001 hasta 2008. En la siguiente figura se puede observar la distribución por años y por tipo de trabajo. 
Fig. 10. Distribución por año y por tipo de trabajo

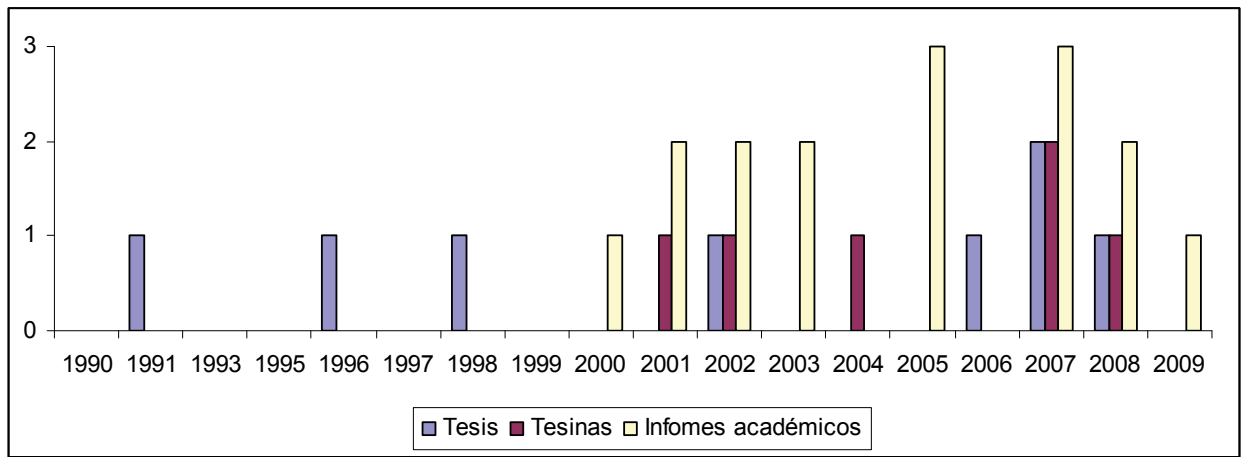

\section{CONCLUSIONES}

En primer lugar nos ha llamado la atención la falta de unanimidad por parte de los centros docentes a la hora de denominar las titulaciones relacionadas con biblioteconomía y documentación, ocurriendo lo mismo con la denominación correspondiente a la materia de catalogación, así por ejemplo se han utilizado para las titulaciones términos como Licenciatura en Bibliotecología y Ciencias de la Información, Licenciado en Ciencias de la Información Documental, Licenciado en Bibliotecología, Licenciatura en Biblioteconomía, etc., y para la materia de Catalogación, Organización de la información, Códigos de catalogación, Descripción documental, catalogación, etc.

A este respecto opinamos que debería haber un intento de normalización, tanto en la terminología como en los contenidos recogidos en los diferentes planes de estudios, siguiendo el modelo establecido por el Plan Bolonia para Europa.

Monografías:

- Dentro de los años de producción destaca el periodo comprendido entre 2007 y 2008 con un total de catorce monografías publicadas.

- Se observa que la mayoría de los autores, un $80 \%$ son autores únicos siendo el promedio entre el número de autores y las monografías de 1,25.

- En cuanto al tipo de autores destacar que prácticamente la totalidad corresponde a autores personales habiéndose localizado sólo una publicación con autoría corporativa realizada por el Comité Técnico de Normalización Nacional de Documentación.

- En relación al género la mujer continúa siendo la acaparadora en la autoría de los trabajos con un porcentaje del $60 \%$.

- Por lo que se refiere a los lugares de publicación, todas las obras han sido publicadas de México D.F, destacando como entidad editora la Universidad Nacional Autónoma de México (UNAM) con dieciséis publicaciones junto a la Escuela Nacional de Biblioteconomía y Archivonomía con tres y el Instituto 
Latinoamericano de la Comunicación Educativa (ILCE) con dos; por lo que como puede observarse todas las entidades editoras pertenecen al ámbito público.

- Respecto al formato y al idioma todas las monografías localizadas han sido en formato libro, y en idioma español.

- Por lo que se refiere a los descriptores destaca la gran variedad de términos empleados para indizar el mismo contenido, lo que conlleva a nuestro juicio, a una pérdida de información, aunque si nos hemos encontrado, como ya hemos mencionado anteriormente, términos generales y algunos otros más específi$\cos$, lo que nos indica en muchas ocasiones la correcta indización aunque no una correcta normalización de los términos.

Revistas:

- Dentro del apartado de los años de producción, destaca el año 1994 con un total de cuatro artículos publicados. Es significativo observar que durante el período 1990 a 1993 no se ha publicado artículo alguno, así como tampoco entre los años 2002 y 2004.

- Al igual que en las monografías, la autoría individual predomina frente a la autoría colectiva, siendo el promedio entre el número de autores y los trabajos de 1,15 .

- Respecto al género, detectamos una ligera diferencia entre mujeres y hombres ya que estos últimos les superan tan sólo en un 5\%. En relación al idioma el español sigue siendo la lengua dominante.

- En cuanto a la afiliación institucional, destaca la UNAM con un $83 \%$, lo que denota la gran importancia de esta institución en este sector.

- Del apartado de los descriptores podemos destacar términos relacionados con bibliotecas, como información electrónica, reglas y normas, modelo FRBR, aunque el más empleado ha sido el término general de catalogación. En relación al modelo FRBR tan sólo hemos encontrado dos trabajos indizados con este término, aunque muy posiblemente aparecerán en un futuro próximo nuevos estudios ya que se trata de uno de los temas que están generando más literatura en el momento actual.

Congresos:

Indicar que sólo hemos encontrado publicadas las actas relacionadas con tres encuentros específicas de catalogación: Encuentro de Nacional Catalogación y Metadatos (3․ 2008. México, D.F.), Encuentro Internacional de Catalogación ( $2^{\circ}$. 2006. México, D.F.) y Encuentro Nacional de Catalogación y Metadatos ( $2^{\circ}$. 2007. México, D.F.), publicadas entre los años 2007 y 2009. En relación a otros eventos celebrados, ya no específicos de catalogación pero en los que se presentaban comunicaciones relacionadas con esta materia, hemos encontrado cuatro, mencionados en el apartado 3.3., que se celebraron entre los años 1996 y 
2002. Tanto en unos eventos como en otros destaca el CUIB como máximo organizador.

Literatura gris:

Por último destacar la importancia que tanto alumnos como asesores han dado a la catalogación ya que se han presentado, en el periodo estudiado, ocho tesis, seis tesinas y dieciséis informes académicos, lo que a nuestro juicio, denota un elevado interés sobre la materia.

\section{REFERENCIAS BIBLIOGRÁFICAS}

ARRIOLA NAVARRETE, O. Panorama general de la educación bibliotecaria en México. ACIMED, vol.19, (marzo, 2009), nº 3. [online] ISSN 1024-9435.

CLAUSÓ GARCÍA, A. y CARPALLO BAUTISTA, A. Producción científica de las publicaciones españolas referentes al Análisis Documental Formal (ADF) de documentos: 1990-2006. Anales de Documentación, (2007), $\mathrm{n}^{\circ}$ 10, ISSN: 1575-2437, p. 31-48.

GUTIÉRREZ CHIÑAS, A. Identidad profesional de la bibliotecología en México a través de su enseñanza. Investigación Bibliotecológica, vol. 44, (enero/abril, 2008), nº 44, ISSN: 0187-358X, p. 77-87.

SPINAK, E. Diccionario enciclopédico de Bibliometría, Cienciometría e Informetría. Caracas: UNESCO, 1996, 245 p. ISBN: 92-9143-007-2.

VANTI, N. Métodos cuantitativos de evaluación de la ciencia: bibliometría, cienciometría e informetría. Investigación Bibliotecológica, vol. 14, (julio/ diciembre, 2000), n $n^{\circ}$ 29, ISSN: 0187-358X, p. 9-23.

\section{ANEXOS}

a) Listado autores de monografías ordenado alfabéticamente Arreguín Meneses, Sergio

Barquet Téllez, Concepción

Comité Técnico de Normalización Nacional de Documentación (COTENNDOC),

(México)

Escamilla González, Gloria

Fernández de Zamora, Rosa María

Filio López, Hilda Evangelina

García López, Carlos Benigno

Garduño Vera, Roberto

Gorman, Michael

Guevara Villanueva, Angélica

Martínez Arellano, Filiberto Felipe

Mejía Ruiz, Jorge Alberto

Naumis Peña, Catalina 
Popoca Burgoa, Marisol

Rodríguez Gallardo, Adolfo

Romero Tejeda, Emma Norma

Vallejo Hernández, Lourdes Milagros

Vargas Martínez, Celia

\section{Otras responsabilidades:}

Escalona Ríos, Lina, comp.

Escamilla González, Gloria, tr.

Figueroa Alcántara, Hugo Alberto, comp.

Hernández Herrera, Margarita, col.

Lafuente López, Ramiro, pr.

Lugo Hubp, Margarita, coord.

Martínez Arellano, Filiberto Felipe, comp.

Martínez Arellano, Filiberto Felipe, trad. y comp.

Ramírez Velázquez, César Augusto, comp.

Ramos Díaz, María de los Ángeles, col.

Sánchez Luna, Blanca Estela, col.

b) Listado autores de artículos de revista ordenado alfabéticamente

Arriola Navarrete, Oscar

Barrientos Bernabé, Ofelia

Basilio Romero, Concepción

Bautista Flores, Edith

Escalona Ríos, Lina

García López, Carlos

García, Idalia

Garrido Yáñez, Orlanda Angélica

Gómez Briseño, Jorge

Juárez Flores, Crescencio

Kyung-Sun Kim

Lafuente López, Ramiro

Martínez Arellano, Filiberto Felipe

Mosqueda Martínez, Luz María

Pagaza García, Rafael

Quijano Solís, Alvaro

Ramírez Nieto, Alejandro

Ramos Díaz, María de los Angeles

Rodríguez García, Ariel Alejandro

Rodríguez Hernández, Georgina

Rosa Valgañón, Patricia de la

Salgado-Ruelas, Silvia 
Vivas Escobedo, Isaac

c) Relación de entidades a las que están adscritas los autores de los artículos

El Colegio de México

Escuela Nacional de Biblioteconomía y Archivonomía

Instituto Nacional de Antropología e Historia

UNAM. CUIB

UNAM. Dirección General de Bibliotecas

UNAM. Facultad de Filosofía y Letras

UNAM. Instituto de Geología

UNAM. Instituto de Investigaciones Históricas

d) Relación de los títulos de las revistas analizadas

Alquimia

Biblioteca universitaria

Bibliotecas y archivos (México, D.F.)

Gaceta Bibliográfica. Instituto de Investigaciones Bibliográficas

Hemera: Revista de ciencias bibliotecológica y de la información

Información: producción, comunicación y servicios

Investigación Bibliotecológica: archivonomía, bibliotecología e información

e) Relación de las entidades editoras de las monografías

Consejo Nacional para la Cultura y las Artes (México). Dirección General de Bibliotecas

Escuela Nacional de Biblioteconomía y Archivonomía (México)

Información Científica Internacional

Instituto Latinoamericano de la Comunicación Educativa (ILCE)

Red Nacional de Bibliotecas Públicas (México)

Secretaría de Comercio y Fomento Industrial, Dirección General de Normas

Universidad Nacional Autónoma de México. Facultad de Filosofía y Letras

Universidad Nacional Autónoma de México. Instituto de Investigaciones Bibliográficas

Universidad Nacional Autónoma de México. CUIB

Universidad Nacional Autónoma de México. Dirección General de Bibliotecas

Universidad Nacional Autónoma de México. Escuela Nacional de Estudios Profesionales (Acatlán, México (Estado))

Universidad Nacional Autónoma de México. Secretaría de Servicios Académicos

f) Relación de autores y asesores de tesis, tesinas e informes académicos Autores

Aguilar Hernández, Nereyda

Andrade Martínez, Fernando 
Añorve Aguirre, Carlos Daniel

Becerril Galván, Eva

Cruz García, Sandra Araceli

Cruz Rojas, Elisa

Escobar Rodríguez, Antonio

Filio López, Hilda Evangelina

Gante Reyes, Hermilo de

García Villeda, Guillermo Gerardo

González García, Ana Belem

Martínez Gil, Alfredo

Martínez Romero, María Alejandra

Mendoza, Gustavo

Morales Gutiérrez, Verónica

Muñoz Bonilla, Jorge Antonio

Novelo Peña, Raúl

Olguín Hernández, María de Jesús

Ortíz Mar, Alejandro

Peralta Figueroa, Ana Beatriz

Ramírez Ramos, José Carmen

Reséndiz Padrón, Sara

Rodríguez García, Ariel Alejandro

Rodríguez González, Guadalupe de la Cruz

Santana Chavarría, Evelia

Serrano Cruz, Aurora

Valdez Torres, Guillermo

Vega Briones, Zoila Alfonsa, de la

Zavala Botello, María Guadalupe

\section{Asesores}

Ávila Luna, María Longina

Azotla Alvarez, Magdaleno

Barrera Galán, María Guadalupe

Butrón Yañez, Katya

Cabral Vargas, Brenda

Camacho Campos, Carlos

Casa Tirao, Beatríz

Chávez Villa, Micaela

Espinosa Becerril, Isabel

Figueroa Alcántara, Hugo Alberto

Flores Valderrama, Armando

González Hernández, María Claudia

Martínez Arellano, Filiberto Felipe 
Molina Mercado, Esperanza

Noguez Ortíz, Araceli

Pagaza García, Rafael

Ramírez Escarcega, Alejandro

Ramírez Velázquez, César Augusto

Rivero Zambrano, Luis Francisco

Rodríguez Gallardo, Adolfo

Rodríguez García, Ariel Alejandro

Sánchez Luna, Blanca Estela

Torres Vargas, Georgina Araceli

Vázquez Santa Ana, María Gabriela 